\title{
El uso de las TIC en la enseñanza de conceptos geométricos en la educación básica
}

\author{
The Use of ICT in the Teaching of Geometric Concepts in Basic Education
}

O uso das TIC no ensino de conceitos geométricos na educação básica

Francisco Flores Cuevas

Universidad de Guadalajara, Centro Universitario de la Costa, México francisco.fcuevas@academicos.udg.mx https://orcid.org/0000-0002-8874-9918

Claudio Rafael Vásquez Martínez

Universidad de Guadalajara, Centro Universitario de la Costa, México crvasquezm@gmail.com https://orcid.org/0000-0001-6383-270X

Felipe Anastacio González González Universidad Autónoma de Tamaulipas, México

felgonzale@docentes.uat.edu.mx https://orcid.org/0000-0002-1410-8616

\section{Resumen}

El presente trabajo se enfocó en el desarrollo de competencias mediante el uso de las tecnologías de la información y la comunicación (TIC), y de manera particular en el uso de GeoGebra (software educativo) como estrategia de enseñanza-aprendizaje de las matemáticas en alumnos de educación secundaria. El diseño de la investigación fue de carácter cuasiexperimental; permitió inferir las relaciones causales entre variables, una independiente y dos dependientes. La muestra estuvo integrada por 60 estudiantes de dos grupos del grado séptimo del Institución Educativa José Miguel de Restrepo y Puerta, del municipio de Copacabana, Colombia, cuyas edades oscilaban entre los 12 y 15 años, y 


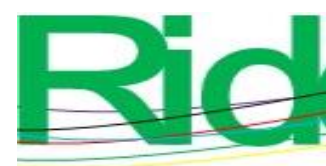

Revista Iberoamericana para la
Investigación y el Desarrollo Educativo
ISSN $2007-7467$

quienes cursaron la asignatura de Matemáticas, curso donde se implementó, para el grupo experimental, el uso del paquete informático GeoGebra y otras herramientas informáticas. Entre los resultados destaca la valía de las TIC para la enseñanza de conceptos geométricos y el desarrollo del pensamiento espacial, así como para que los estudiantes adquieran competencias necesarias para la vida.

Palabras clave: conceptos geométricos, enseñanza de las matemáticas, estrategia didáctica, tecnologías de la información y comunicación.

\begin{abstract}
This work focused on the development of competencies using information and communication technologies (TIC), and in a particular way on the use of GeoGebra (educational software) as a teaching-learning strategy of mathematics in secondary education students. The research design was quasi-experimental in nature; it allowed inferring the causal relationships between variables, one independent and two dependents. The sample was made up of 60 students from two groups of the seventh grade of the José Miguel de Restrepo y Puerta Educational Institution, in the municipality of Copacabana, Colombia, whose ages ranged between 12 and 15 years, and who took the subject of Mathematics, a course where the GeoGebra software package and other IT tools were implemented for the experimental group. Among the results stands out the value of ICT for teaching geometric concepts and the development of spatial thinking, as well as for students to acquire skills necessary for life.
\end{abstract}

Keywords: geometric concepts, mathematics teaching, didactic strategy, information and communication technologies. 


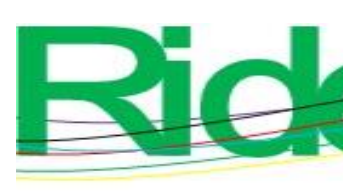

Revista Iberoamericana para la Investigación y el Desarrollo Educativo ISSN 2007 - 7467

\section{Resumo}

Este trabalho centrou-se no desenvolvimento de competências através da utilização de tecnologias de informação e comunicação (TIC) e, de forma particular, na utilização do GeoGebra (software educacional) como estratégia de ensino-aprendizagem de matemática no ensino secundário de alunos. O desenho da pesquisa era de natureza quase experimental; permitiu inferir as relações causais entre as variáveis, uma independente e duas dependentes. A amostra foi composta por 60 alunos de duas turmas da sétima série da Instituição de Ensino José Miguel de Restrepo y Puerta, do município de Copacabana, Colômbia, com idades variando entre 12 e 15 anos, e que cursaram a disciplina de Matemática , um curso onde foi implementado o uso do pacote de software GeoGebra e outras ferramentas de TI para o grupo experimental. Entre os resultados destaca-se o valor das TIC para o ensino de conceitos geométricos e o desenvolvimento do pensamento espacial, bem como para que os alunos adquiram competências necessárias à vida.

Palavras-chave: conceitos geométricos, ensino de matemática, estratégia didática, tecnologias de informação e comunicação.

Fecha Recepción: Enero $2021 \quad$ Fecha Aceptación: Julio 2021

\section{Introducción}

El presente trabajo se enfoca en el desarrollo de las competencias mediante el uso de las tecnologías de la información y la comunicación (TIC), y de manera particular en el uso de GeoGebra (software educativo) como estrategia de enseñanza-aprendizaje de las matemáticas en alumnos de educación secundaria. El uso de esta herramienta digital por parte del docente tiene como objetivo proyectar dinámicas para mejorar el desempeño de los estudiantes y propiciar aprendizajes significativos a corto, mediano y largo plazo. Se trata de un tipo de herramienta que por sí sola resulta una fuente de motivación para los estudiantes pertenecientes al nivel básico de estudios.

La pregunta de investigación se formuló en los siguientes términos: ¿qué impacto tiene el uso de las TIC en los procesos educativos del campo de la geometría? Y de esta pregunta se desprendieron los siguientes cuestionamientos secundarios: ¿cómo se contrapone la enseñanza tradicional de las figuras geométricas y sus transformaciones a una formación respaldada en el uso de las TIC? ¿Qué herramienta informática se debe de aplicar como estrategia didáctica para ayudar al estudiante a hacer modelaciones geométricas? ¿Cuál es la 


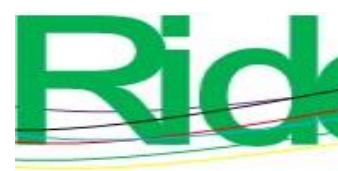

Revista Iberoamericana para la
Investigación y el Desarrollo Educativo
ISSN $2007-7467$

importancia de las TIC en el aprendizaje significativo de los estudiantes de los primeros grados de educación básica y en el mejoramiento de los procesos de comprensión geométrica?

A partir del diseño de estas preguntas de investigación se planteó la siguiente hipótesis: la enseñanza de las figuras geométricas y sus transformaciones en el plano cartesiano han sido infructuosas por la carencia de estrategias pedagógicos apoyadas en herramientas digitales por parte del docente que conlleven a la modelación de cada uno de los procesos de enseñanza-aprendizaje, así como a la adquisición de competencias que contribuyan al aprendizaje significativo por parte de los estudiantes dentro del aula de clase.

\section{Antecedentes y justificación}

Quintero y Costas (1994) afirman:

La forma de enseñar una materia depende, en gran medida, de la visión que tenemos de ella. Generalmente la geometría se asocia con el pensamiento deductivo, exacto, preciso. Dada esta forma de ver la geometría, la enseñanza de esta se dedica mayormente a presentar los resultados en forma deductiva y rigurosa (p. XI).

Para Cabero y Duarte (1999), las TIC permiten una mejor interacción entre docentes y alumnos. Asimismo, facilitan la adquisición de nuevos conocimientos y enriquecen el proceso de enseñanza-aprendizaje con imágenes, videos, audios y otros elementos de multimedia (pp. 23-45).

\section{Referentes teórico-contextuales}

A partir de lo dicho hasta aquí se puede deducir que las bases teóricas de la presente investigación son los procesos de enseñanza-aprendizaje de las matemáticas, el uso de las TIC como estrategias para generar aprendizajes significativos, así como la formación de competencias matemáticas en los estudiantes de educación básica. 


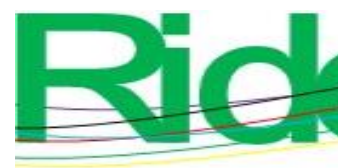

Revista Iberoamericana para la
Investigación y el Desarrollo Educativo
ISSN $2007-7467$

\section{Conceptos geométricos}

La geometría se constituye como una disciplina muy amplia dentro de las matemáticas. Se trata de una rama que presta gran importancia al estudio de las figuras geométricas planas, las cuales suelen ser ordenadas según la forma de sus líneas, ya sean curvas o rectas. Así, por ejemplo, las figuras cónicas son aquellas que están delimitadas por una línea curva cerrada producto de la intersección entre un cono y un plano, tales como el círculo y la elipse. También traza una diferencia entre acciones fundamentales como son mostrar y hacer, observar y actuar, simbolizar y contextualizar e incluye el estudio de factores tales como el plano, el punto, la línea, la recta, la curva, la quebrada, la superficie, el segmento y otros de cuya combinación nacen todas las figuras geométricas, sin olvidar que estas incluyen lados, caras, semejanzas, congruencias, transformaciones, elementos necesarios para un mundo real, lleno de formas bidimensionales y tridimensionales. Es por ello por lo que la geometría debe ser concebida como el aprendizaje de la organización y la comprensión del espacio a través de modelaciones y experiencias constructivas.

Respecto al pensamiento espacial, Giraldo y Ruiz (2014) mencionan lo siguiente:

El pensamiento espacial definido en los lineamientos curriculares como el conjunto de los procesos cognitivos mediante los cuales se construyen y se manipulan las representaciones mentales de los objetos del espacio, las relaciones entre ellos, sus transformaciones y sus diversas traducciones o representaciones materiales, constituye un componente indispensable del pensamiento matemático, hace referencia a la percepción, intuitiva o racional, del entorno propio y de los objetos que hay en él (p. 18).

cuanto a las transformaciones geométricas, Crouch y Haines (2004) mencionan:

En la vida cotidiana constantemente se realizan movimientos y transformaciones en espacios determinados como desplazamientos en bicicletas, rotaciones al girar el cuerpo, observarse en un espejo. Todos los anteriores son ejemplos claros de la practicidad de la geometría en el mundo real de los sujetos. Las transformaciones geométricas son una aplicación del plano en el plano tal que a cada punto de un plano le hace corresponder otro punto del mismo plano (pp. 197-206). 


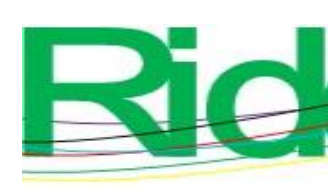

Revista Iberoamericana para la
Investigación y el Desarrollo Educativo
ISSN $2007-7467$

\section{Enseñanza de las matemáticas}

[Las matemáticas son] una disciplina central no solo en nuestra ciencia y nuestro sistema educativo, sino también en todo nuestro universo cultural. Tienen múltiples implicaciones para múltiples aspectos de la sociedad moderna: teorías científicas, desarrollos tecnológicos, análisis económicos y sociopolíticos, etcétera (Díaz y Martins, 1982, pp. 368-373).

Las matemáticas convocan desde cualquier ángulo la significación de algo, y ello está ligado a la formación de todo lo que en el universo se encuentra, pues estas no están asociadas solo con la cantidad, sino que, a través de los tiempos, se ha logrado dar una concepción desde diferentes sistemas y pensamientos. Son útiles para comprender medidas, interpretar situaciones, modelar conceptos, identificar procesos y hasta interpretar informaciones.

Otro aspecto a destacar es la relación del proceso de enseñanza-aprendizaje de las matemáticas con las TIC. De acuerdo con Bartolomé (1999, citado en Sarmiento, 2004), "la utilización de los programas informáticos supone la adquisición de un determinado aprendizaje, pero cabe acotar que es el uso que el docente hace de ellos lo que determina su potencialidad instructiva" (p. 88).

Un docente puede tener un óptimo manejo de los tópicos generativos del área, pero si no posee una metodología que le permita llegar a los alumnos en vano realiza su labor. Para enseñar, es necesario tener claridad frente a lo que los estudiantes requieren, sus intereses por el conocimiento, condiciones para estudiar, nivel de atención, entorno cultural y social en que se desenvuelven, destrezas que puedan tener en el uso de las herramientas TIC y acceso a estas.

Andee Rubin (citado en López, 2007) plantea cinco categorías de herramientas para crear ambientes de aprendizaje enriquecidos por la tecnología: "conexiones dinámicas; herramientas avanzadas; comunidades ricas en recursos matemáticos; herramientas de diseño y construcción; y herramientas para explorar complejidad " (p. 45).

\section{Estrategia didáctica}

Todo individuo requiere, desde su concepción, de un proceso de formación que le permita acatar las normas establecidas del mundo que le circunda y cumplir con las exigencias de la vida social que a lo largo de su existencia deberá asumir. Y si bien la primera etapa de formación corresponde al contacto con sus padres y personas cercanas, es la escuela 


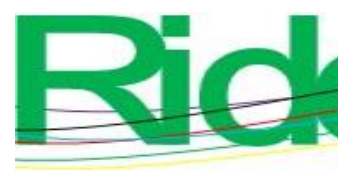

Revista Iberoamericana para la Investigación y el Desarrollo Educativo ISSN 2007-7467

un elemento fundamental en la formación de competencias. "En la escuela se le proporciona a la persona una educación, pero también una formación, una determinada concepción ante la patria, ante los mayores, ante el trabajo y ante la vida" (Piña, y Pontón, 2002, p. 65).

A través de los tiempos, los docentes han brindado un conocimiento de la matemática en muchos casos copiado de modelos que otros les brindaron y que implican solo el cuaderno y el tablero, donde en poco o nada se involucran procesos metacognitivos.

En el aprendizaje de las matemáticas, los niños deben "pensar, formar y reelaborar esquemas o estructuras de conocimientos matemáticos", como lo indican Hernández y Soriano (1999, p. 27), y para ello deben usar procesos cognitivos tales como observar, comparar, ordenar, clasificar, representar, retener, recuperar, interpretar, inferir, evaluar y transferir (Sarmiento, 2004, p. 88).

Lo anterior sugiere que es necesario que las matemáticas sean impartidas como un área integral. "Cuanto más abstracto es un tema más sencillo resulta lograr la integración” (Martinello y Cook, 2000, p. 91, citados en Sarmiento, 2004, p. 106). Dicha integración es la combinación perfecta para potenciar competencias matemáticas, y para la vida a través de las inteligencias múltiples y el pensamiento crítico.

\section{Enseñanza y aprendizaje}

La enseñanza y el aprendizaje son dos procesos que, si bien se conciben como uno solo, son distintos. Por tradición, la enseñanza se reconoce como una acción propia del docente; y el aprendizaje, del estudiante. La enseñanza es la metodología que el docente usa en sus clases para entregar el conocimiento a sus estudiantes. Durante muchos años, el proceso de enseñanza siempre ha estado ligado a la manera en que el docente aborda las temáticas de clase, que, por lo general, obedece a procesos poco didácticos y más memorísticos.

"Enseñar es provocar dinámicas y situaciones en las que pueda darse el proceso de aprender en los alumnos". Entonces, una de las características esenciales de la enseñanza es la intencionalidad. El reto (...) será lograr que aquellos [los alumnos] sean capaces de darle sentido a su conocimiento para que pueda ser utilizado para sus propios fines, y no solo para fines escolares (González, 2008, p. 29). 


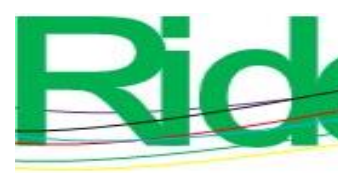

Revista Iberoamericana para la Investigación y el Desarrollo Educativo ISSN 2007-7467

Las estrategias de aprendizaje, entonces, son un cúmulo de actividades, técnicas y medios que se planifican y que tienen como meta satisfacer las necesidades de los estudiantes, los objetivos que se buscan y la naturaleza de los conocimientos. A la hora de definir las estrategias de aprendizaje, el docente debe tener en claro la meta de la clase, cómo quiere enseñar, cómo concibe el aprendizaje, pues tienen gran influencia en el grado de motivación de los estudiantes al aprender.

El objeto formal de la didáctica es "la actividad docente-discente con los métodos adecuados". Otra manera de denominar el proceso comunicativo, bidireccional que tiene lugar en cada acto didáctico. $\mathrm{O}$, dicho de otro modo: el proceso de enseñanza-aprendizaje que acontece cuando están en relación un docente y un discente (o más de uno) en la que el primero selecciona y utiliza diversos procedimientos, métodos o estrategias para ayudar a conseguir el aprendizaje del segundo (Crisanto, 2019, p. 5).

\section{Tecnologías de la información y comunicación}

Existen múltiples definiciones de las TIC. Una de ellas, la de Cabero (1998), señala:

En líneas generales, podríamos decir que las tecnologías de la información y comunicación son las que giran en torno a tres medios básicos: la informática, la microelectrónica y las telecomunicaciones; pero giran no solo de forma aislada, sino lo que es más significativo, de manera interactiva e interconexionadas, lo que permite conseguir nuevas realidades comunicativas (p. 197-206).

Para Bartolomé (citado en Belloch, s. f.), las TIC "encuentran su papel como una especialización dentro del ámbito de la didáctica y de otras ciencias aplicadas de la educación, (...) especialmente [en el] diseño, desarrollo y aplicación de recursos en procesos educativos" (p. 1).

Según la Organización para la Cooperación y el Desarrollo Económicos [OCDE] (2002, citada en Baelo y Cantón, 2009), las TIC son "aquellos medios y servicios que permiten recopilar, almacenar y transmitir información con medios electrónicos” (p. 10). Y Aliaga y Bartolomé (2006) añaden: 


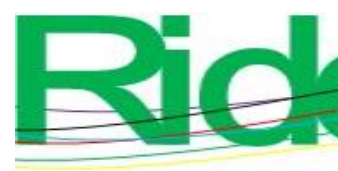

Revista Iberoamericana para la Investigación y el Desarrollo Educativo ISSN $2007-7467$

[Las] TIC han tenido distintos hitos históricos en su desarrollo e implantación social, destacando entre los últimos (aunque ni mucho menos los únicos, como veremos) los acontecidos durante la denominada "revolución digital", especialmente los que hacen referencia a la difusión de los ordenadores personales y, más recientemente, de internet. En cualquier caso, ha de tenerse presente que la tecnología ha de concebirse más bien como un continuo que va desde los libros o las pizarras, pasando por la radio o el video, hasta los elementos informáticos o las aplicaciones de internet más avanzadas (pp. 5588).

Aviram (2002, citado en Marqués, 2012) identifica tres posibles reacciones de los centros docentes para adaptarse a las TIC y al nuevo contexto cultural:

- $\quad$ Escenario tecnócrata: las escuelas se adaptan realizando simplemente pequeños ajustes: en primer lugar la introducción de la "alfabetización digital" de los estudiantes en el currículo para que utilicen las TIC como instrumento para mejorar la productividad en el proceso de la información y luego progresivamente la utilización de las TIC como fuente de información y proveedor de materiales didácticos (aprender de las TIC).

- Escenario reformista: se dan los tres niveles de integración de las TIC que apunta Patiño (2014): los dos anteriores (aprender sobre las TIC y aprender de las TIC) y además se introducen en las prácticas docentes nuevos métodos de enseñanza/aprendizaje constructivistas que contemplan el uso de las TIC como instrumento cognitivo (aprender con las TIC) y para la realización de actividades interdisciplinarias y colaborativas

- Escenario holístico: los centros llevan a cabo una profunda reestructuración de todos sus elementos. La escuela y el sistema educativo no solamente tienen que enseñar las tecnologías, (...) sino que estas aparte de producir unos cambios en la escuela generan un cambio en el entorno" (Marqués, 2012, p. 3).

La escuela es uno de los principales agentes educativos, también está envuelta en todo este maremágnum que caracteriza a la sociedad de la información.

Siguiendo todo lo anteriormente mencionado, las TIC son de gran apoyo como herramientas dentro de la enseñanza y el aprendizaje. La vida actual de los seres humanos ha 


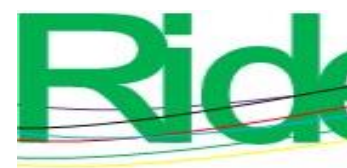

Revista Iberoamericana para la
Investigación y el Desarrollo Educativo
ISSN $2007-7467$

presentado grandes transformaciones que obedecen a las TIC, las cuales brindan la posibilidad de estar interconectados, independientemente de la ubicación en que se encuentren, lo cual acerca a las personas entre sí y con su trabajo, estudio, hogar, entre otros.

Ahora bien, generalmente, las matemáticas son enseñadas separadamente, de acuerdo con los pensamientos y sistemas y no como un todo coherente, con estrategias por parte del docente poco llamativas y tradicionales, dejando de lado los intereses y necesidades del alumno. Esta condición hace ineludible fomentar acciones creativas que seduzcan a los estudiantes en el aprendizaje de las matemáticas. Las herramientas tecnológicas y los programas informáticos fomentan el pensamiento crítico, la adquisición de competencias para la vida, la potenciación de inteligencias múltiples y el aprendizaje significativo.

En esa línea, uno de los softwares más comunes en el área de matemáticas es GeoGebra, un programa didáctico que contribuye a la enseñanza y aprendizaje de las matemáticas en todos los niveles de la educación básica y media, combinando en sus actividades la geometría, el álgebra, el análisis y la estadística. GeoGebra contiene numerosas construcciones geométricas que facilitan el aprendizaje de la geometría.

\section{Metodología}

La investigación presente es de corte transversal y recurre a una metodología cuantitativa, con un diseño de estudio cuasiexperimental. Igualmente, es longitudinal, prospectiva con tres medidas: pretest, test y postest, y compara dos grupos: uno de control y otro experimental.

Este diseño se basa en el supuesto de que la variación de una a otra medida se debe al influjo de la variable experimental, lo que quedará directamente evidenciado en los análisis del grupo control y del grupo experimental (Sierra, 2003).

\section{Muestra}

Este proyecto de investigación es una apuesta por lograr una formación integral donde se conjuguen competencias matemáticas para la vida. Es importante recalcar que la investigación fue posible no solo por la colaboración de los distintos compañeros y compañeras docentes, sino también de las directivas del plantel, quienes amablemente abrieron las puertas y facilitaron los tiempos para implementar el instrumento investigativo. 

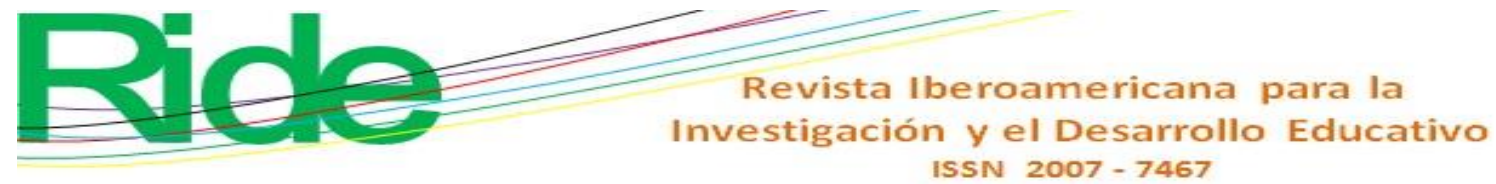

En las siguientes tablas se describe la población de estudiantes en cuanto a género (masculino y femenino) de los grupos control y experimental.

Tabla 1. Población y género de los alumnos del grupo control

\begin{tabular}{|c|c|c|}
\hline Población & Total & $\%$ \\
\hline Alumnos & 30 & 100 \\
\hline Hombres & 16 & 53.3 \\
\hline Mujeres & 14 & 46.6 \\
\hline
\end{tabular}

Fuente: Elaboración propia

Tabla 2. Población y género de los alumnos del grupo experimental

\begin{tabular}{|c|c|c|}
\hline Población & Total & $\%$ \\
\hline Alumnos & 30 & 100 \\
\hline Hombres & 18 & 60 \\
\hline Mujeres & 12 & 40 \\
\hline
\end{tabular}

Fuente: Elaboración propia

\section{Descripción del instrumento}

Esta investigación se enfocó en encuestar a una muestra de 60 estudiantes del grado séptimo de secundaria, cuyas edades oscilaban entre los 12 y 15 años, y quienes presentaban diversos niveles de competencias matemáticas. Las aseveraciones se hicieron con base en los aspectos pedagógicos de los docentes, así como en el uso de las TIC para que mejoren cada uno de sus procesos de enseñanza-aprendizaje dentro de su actividad académica en la Institución Educativa José Miguel de Restrepo y Puerta, del municipio de Copacabana, Colombia. Inicialmente, la encuesta solicita algunos datos básicos a los participantes, pero importantes a la hora de comprender lo que luego se pregunta.

Una encuesta o pretest se realizó a la totalidad de la muestra (grupo experimental y de control); allí se indagó sobre la concepción de la geometría, las herramientas usadas en la construcción y transformación de figuras y sobre el uso de las TIC en cada uno de los procesos de enseñanza-aprendizaje por parte del docente. Posteriormente, se llevó a cabo una segunda encuesta, un postest, a los estudiantes del grupo experimental con la finalidad de 


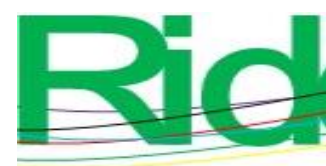

Revista Iberoamericana para la
Investigación y el Desarrollo Educativo
ISSN $2007-7467$

evaluar la experiencia educativa, cómo consideraron la metodología de enseñanza del docente, qué mediadores usaron en las prácticas y qué opinión tienen frente al uso de las TIC.

\section{Técnicas para la recolección de datos}

Las técnicas e instrumentos son los elementos que permiten recopilar la información adecuada y necesaria para realizar cualquier tipo de investigación. Estos incluyen principalmente encuestas, cuestionarios y documentos oficiales, así como textos de autores que hayan desarrollado alguna temática acerca del asunto central. En concreto, la revisión se enfoca en determinar qué tanto los docentes seleccionados utilizan y dominan las TIC dentro de los procesos de enseñanza-aprendizaje en ámbitos rurales de la región del Tequendama (Colombia).

\section{Resultados}

Se podría decir que los métodos aplicados fueron acertados, pues dan cuenta de lo que piensan los estudiantes antes y después de la experiencia, además generan en el docente una necesidad de cualificarse frente a las TIC, para empatizar metodológicamente con los estudiantes, pues como se percibe en la gráficas, los educandos requieren de manera apremiante tener aprendizajes que los atraigan y los vinculen al mundo real, máxime cuando la institución educativa posee los recursos para optimizar la labor.

La implementación del programa de cómputo GeoGebra permite que los estudiantes realicen modelaciones geométricas de manera precisa y tengan mayor habilidad en el uso de herramientas informáticas; así, logran romper con el esquema tradicional de una geometría plana, cuando el mundo que circunda la escuela posee varias dimensiones. Por todo ello, los estudiantes aplauden que el aula de clase sea un laboratorio experimental que mejore la comprensión de la realidad y contribuya a establecer soluciones frente a situaciones del sistema geométrico. 


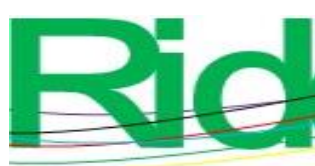

Revista Iberoamericana para la Investigación y el Desarrollo Educativo ISSN 2007 - 7467

Tabla 3. Rango de edades y género de los alumnos del grupo control

\begin{tabular}{|l|c|c|c|c|c|c|c|c|}
\hline \multicolumn{1}{|c|}{ Edades } & 12 & $\%$ & 13 & $\%$ & 14 & $\%$ & 15 & $\%$ \\
\hline Mujeres & 8 & 57.1 & 5 & 35.7 & 1 & 7.1 & 0 & 0 \\
\hline Hombres & 7 & 43.7 & 6 & 37.5 & 1 & 6.25 & 2 & 12.5 \\
\hline Total & 15 & 100 & 11 & 100 & 2 & 100 & 2 & 100 \\
\hline
\end{tabular}

Fuente: Elaboración propia

Figura 1. Rango de edades y género de los alumnos del grupo control

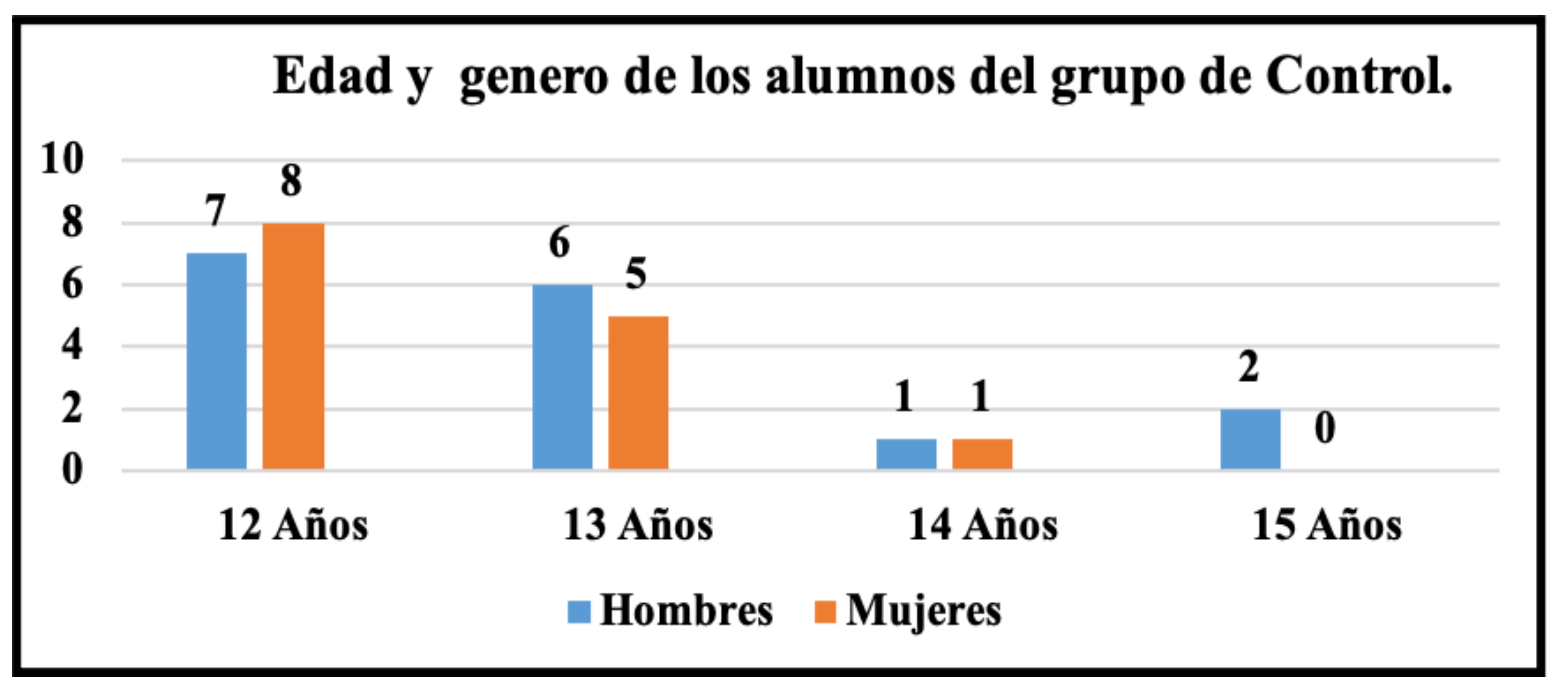

Fuente: Elaboración propia

La figura 1 permite comprender que los estudiantes, en su mayoría, se encuentran en edades regulares para el grado escolar en curso, y además que las mujeres tienen menos edad, pues hay estudiantes que han tenido repitencias, discapacidad o ingresaron de manera tardía a la formación escolar y obedecen más al género masculino. 
Tabla 4. Rango de edades y género de los alumnos del grupo experimental

\begin{tabular}{|l|c|c|c|c|c|c|c|c|}
\hline Edades & 12 & $\%$ & 13 & $\%$ & 14 & $\%$ & 15 & $\%$ \\
\hline Mujeres & 9 & 75 & 3 & 25 & 0 & 0 & 0 & 0 \\
\hline Hombres & 11 & 61.1 & 5 & 27.7 & 1 & 5.5 & 1 & 5.5 \\
\hline Total & 20 & 100 & 8 & 100 & 1 & 5.5 & 1 & 100 \\
\hline
\end{tabular}

Fuente: Elaboración propia

Figura 2. Rango de edades y género de los alumnos del grupo experimental

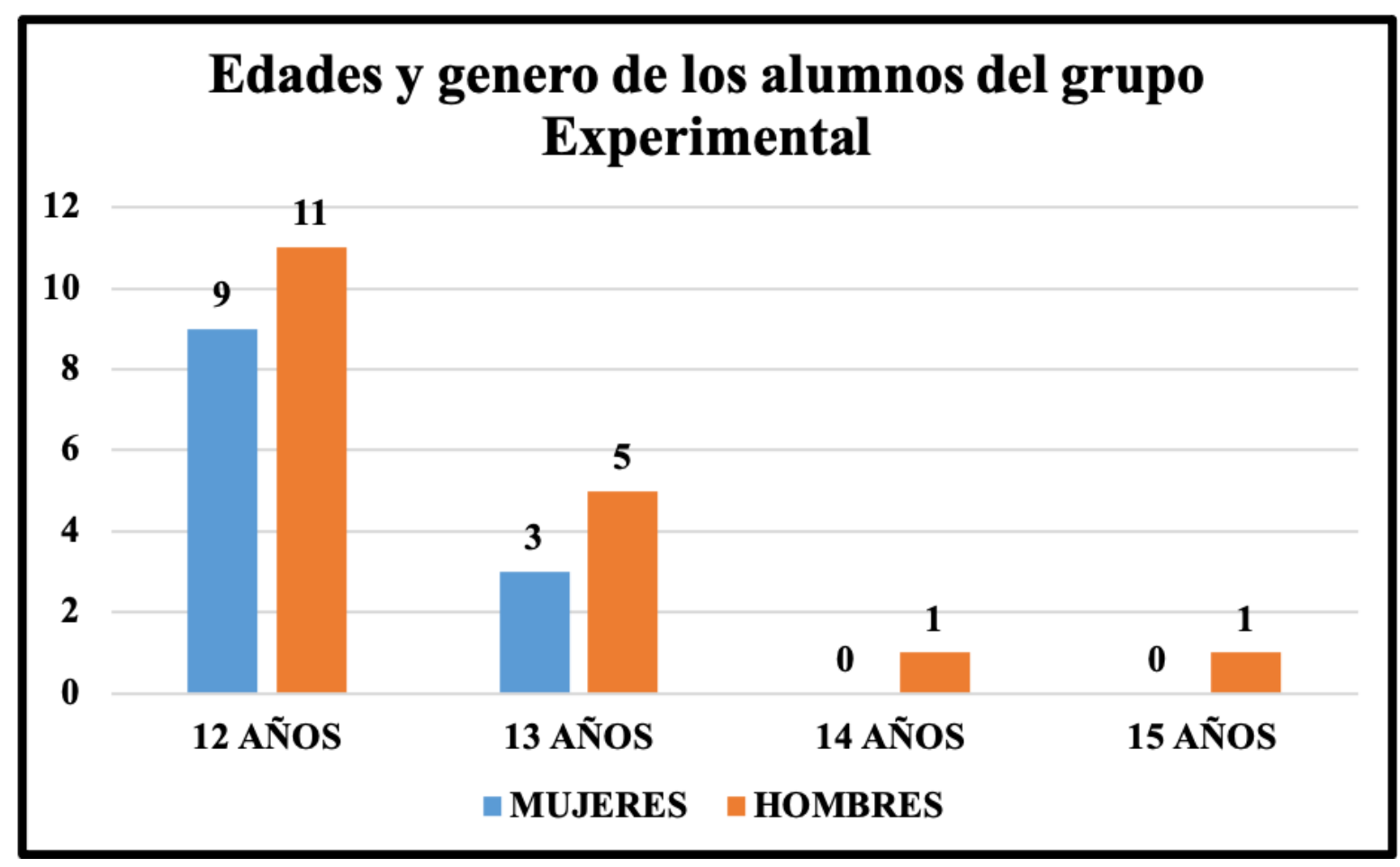

Fuente: Elaboración propia

En la anterior figura se percibe que en el grupo experimental hay más hombres que mujeres. Estas ultimas, a su vez, poseen edades menores y más acordes al grado en curso, pues los estudiantes que tienen entre 14 y 15 años son de sexo masculino y ello es porque han reprobado o presentan alguna discapacidad. 


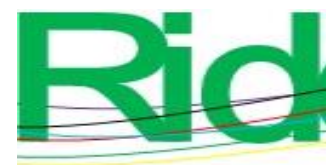

Revista Iberoamericana para la
Investigación y el Desarrollo Educativo
ISSN $2007-7467$

educación básica primaria y el preescolar, pues sus saberes son específicos en un área y pocas veces las matemáticas son su fuerte, por lo cual limitan la enseñanza al sistema numérico y solo brindan temáticas basadas en las principales figuras, sin introducirse en los elementos importantes que las componen, y mucho menos sin hacer construcciones prácticas que permitan la significación de estos elementos de la geometría y otras cosas importantes en relación con las formas.

Figura 4. Análisis de la pregunta dos

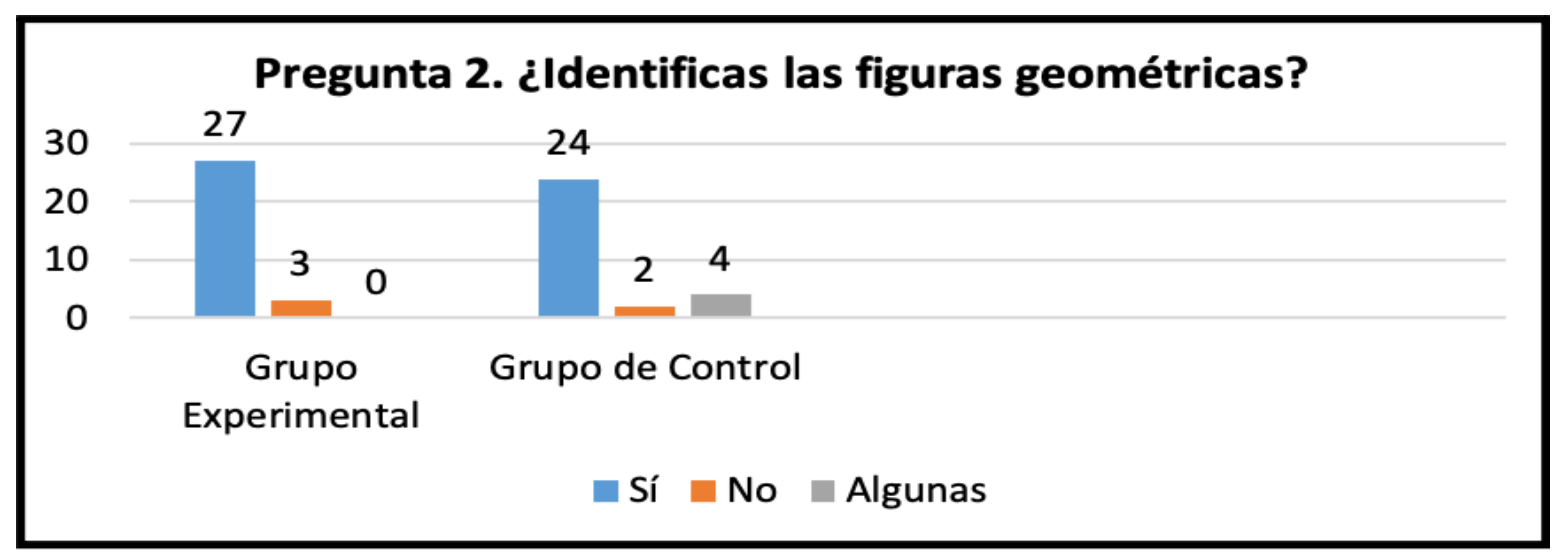

Fuente: Elaboración propia

Al preguntar si identifican las figuras geométricas, los estudiantes de ambos grupos, en su mayoría, respondieron de manera positiva, teniendo en cuenta que para ellos las figuras se reducen a círculo, cuadrado, rectángulo y triángulo, las demás no las asocian con la geometría. Es comprensible teniendo en cuenta que el acercamiento con estas se ha limitado a lo plano y son las figuras con menos lados, lo cual facilita su dibujo o su percepción en formas simples de los objetos del aula. 


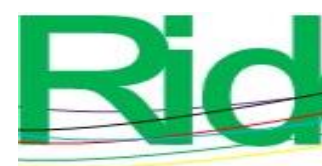

Revista Iberoamericana para la Investigación y el Desarrollo Educativo ISSN 2007-7467

Figura 5. Análisis de la pregunta tres

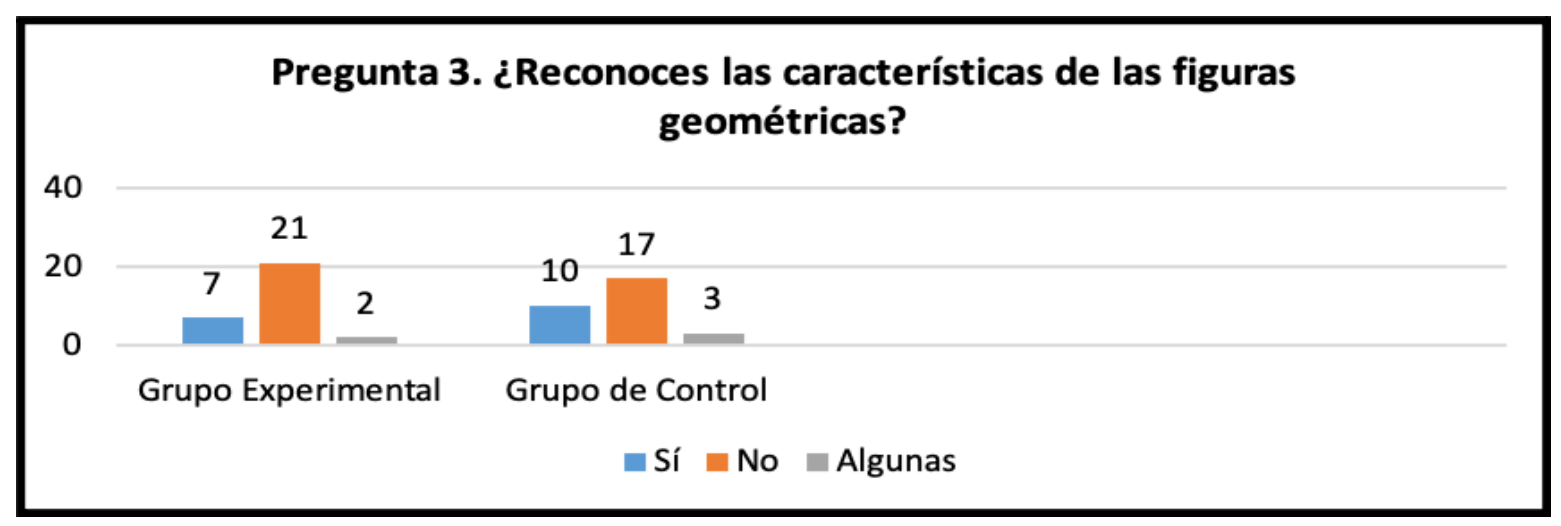

Fuente: Elaboración propia

Tal como lo muestra la figura 5, los estudiantes de ambos grupos, en su mayoría, no reconocen las características de las figuras geométricas. Si bien reconocen el número de lados en las básicas, en las figuras tridimensionales no tienen nociones de lados, aristas, caras, vértices. Así, se puede inferir que la enseñanza relacionada con la geometría está basada en figuras planas; con las tridimensionales, poco o nada se ha tenido contacto. Se trata de una prueba de que la educación matemática es muy carente en este aspecto y, por lo general, el maestro deja estos temas para el último periodo académico, y se aprende si alcanza el tiempo escolar o se asignan como consultas.

Figura 6: Análisis de la pregunta cuatro

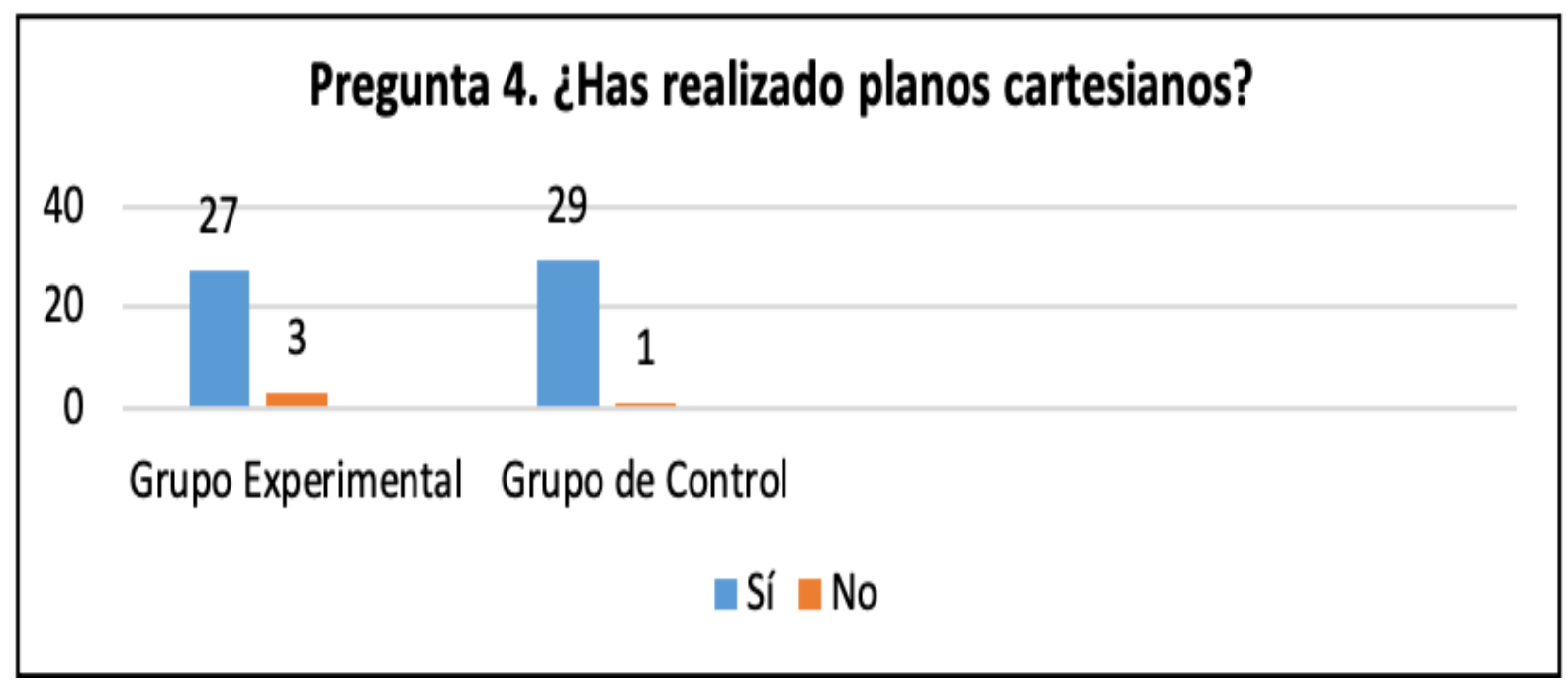

Fuente: Elaboración propia

Los estudiantes afirman que sí han realizado planos cartesianos y, al conversar con ellos, los relacionan con las rectas numéricas y tienen claro los cuadrantes, los ejes positivos 

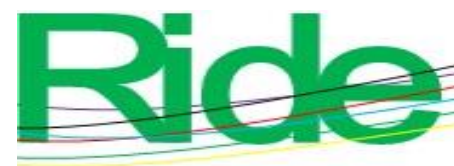

Revista Iberoamericana para la Investigación y el Desarrollo Educativo

ISSN 2007 - 7467

y negativos y sus respectivos nombres. Son claros al enunciar que la mayor dificultad es que sean equidistantes y los aprendieron en el grado anterior, es decir, sexto, donde el docente es licenciado en el área de matemáticas y en la planeación muestra compatibilidad con los derechos básicos de aprendizaje del Ministerio de Educación Nacional (MEN) para este pensamiento y competencias matemáticas.

Figura 7. Análisis de la pregunta cinco

\section{Pregunta 5. ¿Que herramientas has utilizado para la construcción de figuras geométricas?}

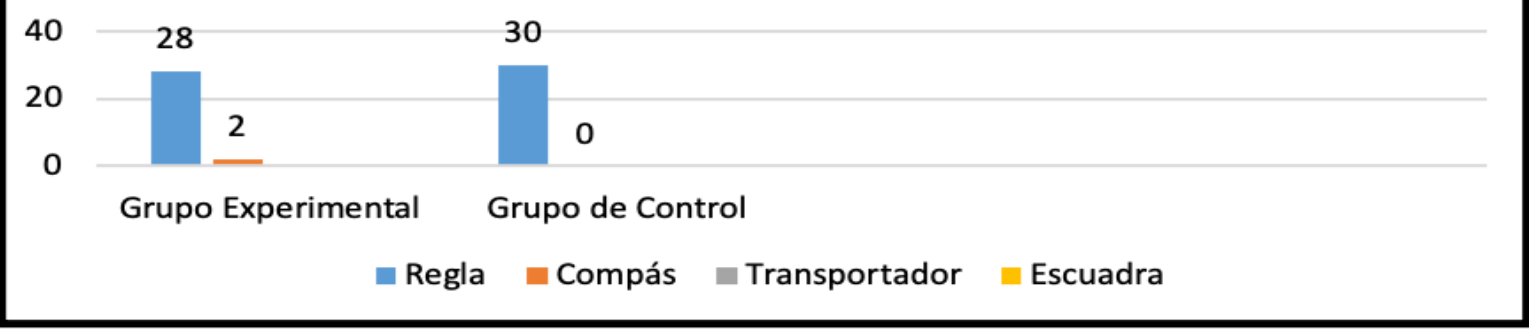

Fuente: Elaboración propia

Como lo mencionan los estudiantes, la herramienta que han usado para la construcción de las figuras geométricas es la regla. De hecho, mencionan que no tienen buen manejo del transportador y del compás y la escuadra solo la usan como instrumento de medición, cuando no poseen regla. En el manejo de la regla se percibe que algunos estudiantes no comienzan el conteo desde cero, sino desde uno, y los trazos en ocasiones no coinciden. Urge, entonces, la manera de hacer construcciones usando otras herramientas. 


\section{Preguntas post intervención para grupo experimental}

Tabla 5. Preguntas post intervención (grupo experimental)

\begin{tabular}{|l|l|l|}
\hline Preguntas postintervención & Sí & No \\
\hline 1) ¿Tienes computador o tableta en tu casa? & 23 & 7 \\
\hline 2) ¿Tienes acceso a internet en tu casa? & 19 & 11 \\
\hline $\begin{array}{l}\text { 3) ¿Consideras que la metodología del docente de matemáticas es } \\
\text { buena? }\end{array}$ & 14 & 16 \\
\hline 4) ¿Conocías el programa “geogebra”? & 17 & 13 \\
\hline 5) ¿Habías utilizado el programa geométrico “geogebra” antes? & 3 & 27 \\
\hline 6) ¿Dónde has usado el programa Geogebra” ...colegio? & 3 & 27 \\
\hline 7) ¿Consideras agradable realizar construcciones con Geogebra? & 30 & 0 \\
\hline 8) ¿Consideras que usar el programa es sencillo? & 30 & 0 \\
\hline 9) ¿Crees que el programa “Geogebra” permite aprender más? & 30 & 0 \\
\hline
\end{tabular}

Fuente: Elaboración propia

La Institución Educativa José Miguel de Restrepo y Puerta cuenta con el mayor número de estudiantes del municipio de Copacabana, Antioquia, debido principalmente a su ubicación privilegiada. Además, está certificada como un colegio de calidad, lo que la hace muy atractiva. Podría afirmarse que los estudiantes y sus familias pertenecen a los estratos de clase media del municipio, por lo que la mayoría de los encuestados, $76 \%$, cuenta con computador o tableta en su casa. Parte su contacto con los medios informáticos es en la institución, donde, si bien se cuenta con estos elementos, se carece de Internet para hacer más eficientes los procesos de enseñanza y aprendizaje.

En el grupo experimental, al aplicar el posintervención, $100 \%$ de los estudiantes expresaron que no tenían conocimientos sobre el programa GeoGebra. Sin embargo, al realizar las actividades prácticas, apoyadas con una metodología activa, el programa en cuestión contribuyó a asimilar de manera comprensiva los aprendizajes y disfrutaron de las clases en el aula. 


\section{Preguntas post intervención para grupo control}

Tabla 6. Preguntas posintervención (grupo de control)

\begin{tabular}{|l|c|c|}
\hline Preguntas posintervención & Sí & No \\
\hline 1) ¿Tienes transportador, regla y compás en tu casa? & 9 & 21 \\
\hline 2) ¿Tienes acceso a textos y material de consulta en tu casa? & 23 & 7 \\
\hline 3) ¿Consideras que la Metodología del docente es aburrida? & 21 & 9 \\
\hline 4) ¿Consideras que la Metodología del docente es diversa? & 1 & 29 \\
\hline 5) ¿Has utilizado herramientas como transportador, regla y compás? & 26 & 4 \\
\hline $\begin{array}{l}\text { 6) ¿Has utilizado de manera creativa otras herramientas para dibujar } \\
\text { figuras planas? }\end{array}$ & 6 & 24 \\
\hline $\begin{array}{l}\text { 7) ¿Consideras agradable realizar construcciones con transportador, } \\
\text { regla y compás? }\end{array}$ & 4 & 26 \\
\hline 8) ¿Consideras que usar estas herramientas es sencillo? & 11 & 19 \\
\hline $\begin{array}{l}\text { 9) ¿Crees que la enseñanza de la geometría plana permite aprender } \\
\text { más? }\end{array}$ & 9 & 21 \\
\hline
\end{tabular}

Fuente: Elaboración propia

La encuesta realizada a los estudiantes del grupo de control, tras la intervención al grupo experimental, da cuenta que luego de realizarse con ellos prácticas geométricas tradicionales, usando herramientas como transportador, regla y compras, limitan la imaginación, la capacidad creadora y, por ende, la significación de los aprendizajes, pues solo se tienen vistas bidimensionales que en nada aportan a la concepción y contacto con el mundo real.

\section{Discusión}

Para responder a la pregunta de esta investigación, la implementación de las TIC en las instituciones educativas tiene gran relevancia en la mejora de la calidad de la educación. Para lograr este objetivo es necesario ir más allá de la sola incorporación de medios tecnológicos en el aula; es imprescindible que los docentes estén en continua preparación y actualización de las herramientas tecnologías, con el objetivo de mejorar las competencias y dominio de cada uno de los contenidos de la asignatura de las matemáticas dentro de los procesos de enseñanza-aprendizaje, y así los alumnos sean capaces de desarrollar las competencias matemáticas, así como la adquisición de aprendizajes significativos. 


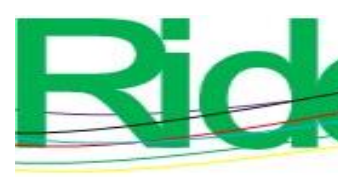

Revista Iberoamericana para la
Investigación y el Desarrollo Educativo
ISSN $2007-7467$

Otro aspecto que da arroja luz sobre la pregunta principal es que la mayoría de los estudiantes no tienen claro el concepto de geometría, simplemente lo asocian a las figuras geométricas. Es un hecho que el pensamiento geométrico es uno de los menos potenciados por los docentes, especialmente los de la educación básica primaria y el preescolar, pues sus saberes son específicos en un área y pocas veces son las matemáticas su fuerte, por lo cual limitan la enseñanza al sistema numérico y solo brindan temáticas basadas en las principales figuras, sin introducirse en los elementos importantes que las componen y mucho menos sin hacer construcciones prácticas que permitan la significación de estos elementos de la geometría y otras cosas importantes en relación con las formas.

En este caso, los esfuerzos para la instrucción de las figuras geométricas y sus transformaciones en el plano cartesiano han sido infructuosos por la carencia de estrategias pedagógicos apoyadas en herramientas digitales por parte del docente que conlleven a la modelación de cada uno de los procesos de enseñanza-aprendizaje, así como a la adquisición de competencias que contribuyan al aprendizaje significativo por parte de los estudiantes dentro del aula de clase.

Cabe mencionar que las disciplinas parten de un acervo muy rico de saberes, cuya enseñanza se encuentra ligada a los conocimientos adquiridos durante un ciclo de vida y a un enfoque social. Esta serie de conocimientos estructurados se les da formalidad dentro de los planes y programas de estudio, los cuales llevan una intención, objetivo y finalidad. La gran pregunta es qué de todos estos conocimientos, habilidades y destrezas deben realmente saber los estudiantes y para qué.

\section{Conclusiones}

La apuesta curricular por una enseñanza de la geometría en la educación básica secundaria aboga por procesos centrados en las necesidades e intereses de los estudiantes y su conexión con el mundo global. Se trata de un desafío necesario: afrontar, en un proceso de resignificación, las nuevas demandas de la sociedad. El estudio realizado le apuesta a la implementación de una estrategia didáctica en un área que muchos consideran tediosa, por lo cual la tarea se centra en promover la creatividad y la motivación; una estrategia para aprender haciendo en los diversos escenarios de la vida del estudiante. 

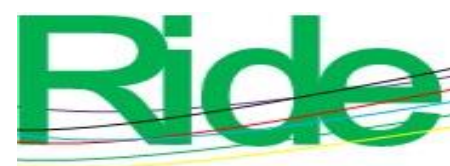

ascenso y crecimiento en su profesión, con conocimientos en el uso y manejo de las TIC; y además de mantener los logros alcanzados y actualizar sus habilidades, también sirven de apoyo a los más noveles en esta profesión.

\section{Contribuciones a futuras líneas de investigación}

Como futuras líneas de investigación se propone la capacitación de los docentes para el uso de herramientas tecnológicas, con la imperiosa necesidad de la implementación de estrategias activas, así como la implementación de diferentes actividades con el apoyo de TIC que inviten a los estudiante de educación básica a la reflexión y la apropiación de conocimientos geométricos específicos. De igual manera, se sugiere que los docentes deben cambiar la manera tradicional de enseñar y hacer uso de los recursos que posee la institución educativa en cuestión, generar acciones didácticas en torno a la informática, teniendo en cuenta que los estudiantes tienen agrado e interés hacia las herramientas tecnológicas.

Finalmente, es necesario que los docentes usen los programas para sus clases de matemáticas, especialmente el software GeoGebra, para así dinamizar los procesos y hacer de la matemática y la geometría un portal al conocimiento y la adquisición de competencias para la vida. 


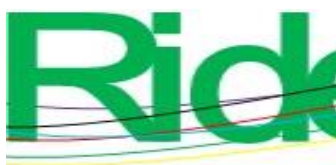
Revista Iberoamericana para la
Investigación y el Desarrollo Educativo
ISSN $2007-7467$

\section{Referencias}

Aliaga, F. y Bartolomé, A. R. (2006). El impacto de las nuevas tecnologías en Educación.En Escudero, T. y Correa, A., Investigación en innovación educativa (pp. 55-88). Madrid, España: La Muralla. Recuperado de http://www.lmi.ub.edu/personal/bartolome/articuloshtml/2006_aliaga_bartolome.pd f.

Alsina, A. (2009). El aprendizaje realista: una contribución de la investigación en Educación Matemática a la formación del profesorado. En González, M. J., González, M. T. y Murillo, J. (eds.), Investigación en educación matemática XIII (pp. 119-127). Santander, España: SEIEM.

Ausubel D., Novak J. y Hanesian H. (2012). Psicología educativa. Un punto de vista cognoscitivo. México: Trillas. (p. 65).

Ávalos, B. y P. Aylwin y Carlson, B. (2005). La inserción laboral de los profesores nuevos en Chile. Paideia, 38, 9-27.

Baelo, R. y Cantón, I. (2009). Las tecnologías de la información y la comunicación en la educación superior. Estudio descriptivo y de revisión. Revista Iberoamericana de Educación, (50). Recuperado de https://rieoei.org/historico/deloslectores/3034Baelo.pdf.(p. 10).

Balacheff N. (2000). Entornos informáticos para la enseñanza de las matemáticas. Complejidad didáctica y expectativas. Educational Studies in Mathematics, 18 (pp. 147-176)

Bartolomé, A. (1999). Nuevas tecnologías en el aula. Guía de supervivencia. Barcelona, España: Graó. (p. 88).

Beck, U. (1998). ¿Qué es la globalización? Falacias del globalismo, respuestas a la globalización. Barcelona, España: Paidós.

Belloch, C. (s. f.). Las tecnologías de la información y la comunicación (TIC) como recurso para la educación. Recuperado de https://www.uv.es/bellochc/pdf/08edu_tema1.pdf. (p. 1).

Beltrán, J. (2003). Enseñar a aprender. México: Educared. (pp. 38-46)

Borrás, I. (2007). Enseñanza y aprendizaje con la Internet: una aproximación crítica. Comunicación y Pedagogía, (151), 28-32. 


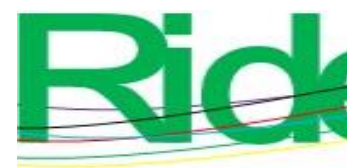

Revista Iberoamericana para la Investigación y el Desarrollo Educativo ISSN $2007-7467$

Cabero, J. (1998). Impacto de las nuevas tecnologías de la información y la comunicación en las organizaciones educativas. En Lorenzo, M. (coord.), Enfoques en la organización y dirección de instituciones educativas formales y no formales (pp. 197-206). Granada, España: Grupo Editorial Universitario

Cabero, J. (2015). Las nuevas tecnologías y las transformaciones de las instituciones educativas. En Lorenzo, M., García, M., Torres, J., Ortega, J., Debón, S. y Ontoria, A. (eds.), Las organizaciones educativas de la sociedad neoliberal (pp. 218-228). Granada, España: Grupo Editorial Universitario.

Cabero, J. y Duarte, A. (1999). Evaluación de medios y materiales de enseñanza en soporte multimedia. Píxel-Bit. Revista de Medios y Educación, (13), 23-45.

Cascallana, M. T. (1988). Iniciación a la Matemática. Materiales y recursos didácticos. Madrid. Santillana, Aula XXI, 1988. (p. 51)

Castells, M. (2008). Internet y la sociedad red. En Lección inaugural del programa de doctorado sobre la sociedad de la información y el conocimiento. Granada: Grupo Editorial Universitario. (pp. 13-24)

Crisanto, A. (2019). Clasificación de la didáctica. Tecnología educativa. Recuperado de https://principiosdedidactica.blogspot.com/2019/11/clasificacion-de-ladidactica.html. (p. 5).

Crouch, R. and Haines, C. (2004). Mathematical modelling: transitions between the real world and the mathematical model. International Journal of Mathematical Education in Science \& Technology, 35(2). (pp. 197-206).

Díaz J, Martins A. (1982). Estrategias de enseñanza aprendizaje. Orientaciones didácticas para la docencia universitaria. Serie de Libros y Materiales Educativos. No.50. San José, Costa Rica: Instituto Interamericano de Cooperación para la Agricultura IICA; 1982. (pp. 368-373).

Ferrer, G. (2006). Estándares en educación. Implicancias para su aplicación en América Latina. Santiago, Chile: Programa de Promoción de la Reforma Educativa en América Latina y el Caribe.

García, B., Loredo, J. y Carranza, G. (2008). Las aproximaciones teórico-metodológicas en los trabajos de la RIED: consideraciones en torno a la construcción de un modelo de evaluación de la práctica docente. En Rueda, M. (ed.), La evaluación de los 

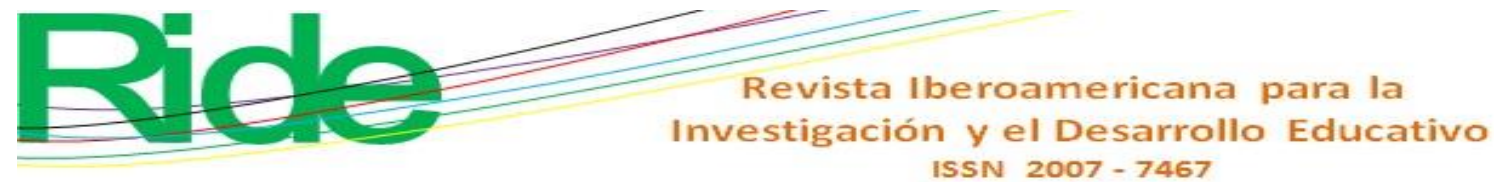

profesores como recurso para mejorar su práctica (pp. 162-220). México: IISUEUNAM-Plaza y Valdés.

García, L. (2004). La modelación matemática en el proceso de enseñanza-aprendizaje del cálculo diferencial. México. Editorial Fondo Tesis.

Gimeno, S. y Pérez, A. (1998). La enseñanza: Su teoría y su práctica. Madrid, España: Akal.

Giraldo, M. L. y Ruiz, M. A. (2014). Aprendizaje significativo del pensamiento espacial y sistemas geométricos, integrando las TIC a través de actividades lúdicas en el primer ciclo de básica. (Tesis de maestría). Universidad Libre - Seccional Cali, Santiago de Cali. (p. 18).

Gisbert M. (2000). El profesor del siglo XXI: de transmisor de contenidos a guía del ciberespacio. Recuperado de https://campus.fahce.unlp.edu.ar/pluginfile.php?file=\%2F122599\%2Fmod_resource $\% 2 \mathrm{Fcontent} \% 2 \mathrm{~F} 1 \% 2 \mathrm{FLa} \% 20$ tecnolog\%C3\%ADa\%20y\%20su\%20repercusi\%C3\% B3n\%20para\%201a\%20tarea\%20de\%20los\%20docentes\%20Las.pdf.

González, V. (2008). Enseñanza y aprendizaje. Ciudad de México, México: Pax. (p. 29).

Gutiérrez, A. (1995). Comprometer al profesorado. Cuadernos de Pedagogía, (241), 31-33.

Hernán, F. y Carrillo, E. (1999). Recursos en el aula de matemáticas. Madrid, España: Síntesis.

Hernández Pina F. Y Soriano E. (1999): Enseñanza y aprendizaje de las Matemáticas en Educación Primaria. Madrid, La Muralla. (p. 27).

Linares, S. y Sánchez, M. V. (1998). Aprender a enseñar matemáticas: Los videos como instrumento metodológico en la formación inicial de profesores. Revista de Enseñanza Universitaria, 13, (pp. 29-44).

Llinares, S. (2011). Tareas matemáticas en la formación de maestros: Caracterizando perspectivas. Números. Revista de Didáctica de las Matemáticas, 78, 5-16.

López, M. (2007). Aplicación de las tecnologías de información para obtener un nivel de aprovechamiento académico satisfactorio en alumnos de tercer semestre del nivel medio superior en la materia de Matemáticas en la Preparatoria Emiliano Zapata. Universidad Autónoma de Nuevo León, San Nicolás de los Garza. (p. 45).

Martinello, M. y Cook, G. (2000). Indagación interdisciplinaria en la enseñanza y el aprendizaje. Editores: Gedisa. País: España. Año de publicación: 2000. (p. 27). 


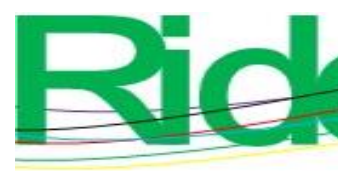

Revista Iberoamericana para la Investigación y el Desarrollo Educativo ISSN $2007-7467$

Marqués, P. (2012). Impacto de las TIC en la educación: funciones y limitaciones. 3ciencias. Recuperado de https://www.3ciencias.com/wp-content/uploads/2013/01/impacto-delas-tic.pdf.

Mendoza, P. y Galvis, A. (1999). Ambientes virtuales de aprendizaje: una metodología para su creación. Información Educativa, 12(2), 295-317.

Morín, E. (2007). Unir los conocimientos. El desafío del siglo XXI. La Paz, Bolivia: Plural.

Organización de las Naciones Unidas para la Educación, la Ciencia y la Cultura [Unesco]. (2008). Normas sobre competencias en TIC para docentes. París, Francia: Organización de las Naciones Unidas para la Educación, la Ciencia y la Cultura. Recuperado de https://www.campuseducacion.com/blog/wpcontent/uploads/2017/02/Normas_UNESCO_sobre_Competencias_en_TIC_para_D ocentes.pdf.

Patiño, C. (2014). Apuntes para una historia de la educación en Colombia. Actualidades Pedagógicas, 1(64). (pp. 261-265).

Piña, J. M. y Pontón, C. E. Coor. (2002). Cultura y Procesos Educativos. Editorial UNAM Primera Edición 2002. (p. 65).

Quintero, A. H. y Costas, N. (1994). Geometría. San Juan, Puerto Rico: Editorial de la Universidad de Puerto Rico. (p. XI).

Salinas J. (1997). Nuevos ambientes de aprendizaje para una sociedad de la información. Revista Pensamiento Educativo, (20), 81-104.

Sarmiento, M. (2004). La enseñanza de las matemáticas y las NTIC. Una estrategia de formación permanente. (Tesis de doctorado). Universitat Rovira i Virgili, Tarragona. (p. 88).

Sierra, R. (2003). Tesis doctorales y trabajos de investigación científica. Madrid, España: Paraninfo.

Vasco, C. (2011). Formación y educación, pedagogía y currículo. En Educación, pedagogía y currículo. Tomo 1 (pp. 15-35). Cali, Colombia: Red Iberoamericana de Pedagogía. 


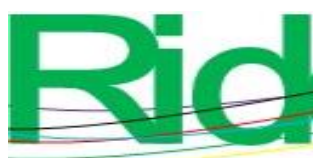

Revista Iberoamericana para la Investigación y el Desarrollo Educativo ISSN 2007-7467

\section{Felipe Anastacio González González}

Doctor en Educación Universidad de Baja California, Maestro en Tecnologías para el Aprendizaje por la Universidad Autonoma de Tamaulipas en Ciudad Victoria, Tamaulipas. Diversos diplomados disciplinares en formación docente por la Universidad Autonoma de Tamaulipas dentro de los programas para el Fortalecimiento Docente. Es catedrático de tiempo completo en la Universidad Autonoma de Tamaulipas, Campus Ciudad Mante, Tamaulipas. Cuenta con Perfil Deseable del Programa de Mejoramiento del Profesorado (Promep), Candidato al Sistema Nacional de Investigadores. Ha publicado libros y varios artículos en revistas nacionales e internacionales. 


\begin{tabular}{|c|c|}
\hline 0 & $\begin{array}{l}\text { Revista Iberoamericana para la } \\
\text { Investigación y el Desarrollo Educativo } \\
\text { ISSN } 2007-7467\end{array}$ \\
\hline Rol de Contribución & Autor (es) \\
\hline Conceptualización & Francisco Flores Cuevas \\
\hline Metodología & Francisco Flores Cuevas \\
\hline Software & Francisco Flores Cuevas \\
\hline Validación & Claudio Rafael Vásquez Martínez \\
\hline Análisis Formal & Claudio Rafael Vásquez Martínez \\
\hline Investigación & Francisco Flores Cuevas \\
\hline Recursos & Francisco Flores Cuevas \\
\hline Curación de datos & Felipe Anastacio González González \\
\hline $\begin{array}{l}\text { Escritura - Preparación del borrador } \\
\text { original }\end{array}$ & Felipe Anastacio González González \\
\hline Escritura - Revisión y edición & Claudio Rafael Vásquez Martínez \\
\hline Visualización & Felipe Anastacio González González \\
\hline Supervisión & Francisco Flores Cuevas \\
\hline Administración de Proyectos & Francisco Flores Cuevas \\
\hline Adquisición de fondos & Francisco Flores Cuevas \\
\hline
\end{tabular}

